\title{
EVALUATION OF THE IMPROVEMENT RP POWDER STEREO-SHAPED TECHNIQUES TO DIRECTLY SOLIDIFIED MODELING OF BIOCHEMISTRY COMPOSITE POWDER MATERIAL
}

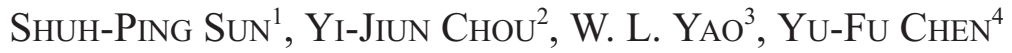 \\ ${ }^{1}$ Department of Biomedical Engineering, I-Shou University, \\ ${ }^{2}$ Department of Orthopaedic Surgery, Kaohsiung Veterans General Hospital, \\ ${ }^{3}$ Department of Mechanical Automation Engineering, National Kaohsing First University of \\ Science and Technology, \\ ${ }^{4}$ Department of Materials Science and Engineering, I-Shou University, Kaohsiung, Taiwan
}

\begin{abstract}
This study apply the RP powder stereo-shaped techniques and using standard biochemistry materials to discuss how biochemistry composite powder material can directly be solidified by using rapid prototyping machines to become bone-filling graft for missing bone on clinical surgery. As for the materials of implants, the research using commercialized composite powder materials. Concerning the making of bone filling model, this study uses 3D Anthropometrics bone image and using the related images editing system to simulate the curve shape of bone filling graft. Then cooperate the characteristic of the rapid prototyping machine (Zcorp 402 3DP), solidify directly without mold, with the commercialized biochemistry bone graft material, it can directly accomplish bone filling graft in the light of patients' demand in the fist place. The forming structure of the rapid prototyping machine is the bone filling piled up by the stratification piling method; it's different from the hand-made pressure forming. According to the modification of the RP equipment, which the research is used, can be upgrade the solidification quality of bone graft material.
\end{abstract}

Biomed Eng Appl Basis Comm, 2006(August); 18: 198-201.

Keywords: rapid prototyping; composite bone graft material

\section{INTRODUCTION}

Due to the mutually of reverse engineering technology and the rapid prototyping production

Received: Feb. 27, 2006; Accepted: June 12, 2006

Correspondence: Yi-Jiun Chou, M.D.

Department of Orthopaedic Surgery, Kaohsiung Veterans General Hospital, 386 Dajung 1st Rd.

Kaohsiung, Taiwan

E-mail: yjchou@isca.vghks.gov.tw method could combines CAD and CAM. It not only can apply in products design but also can using in clinical surgery that could promote the operation quality and shorten the preparation time [1-3]. Due to the reverse engineering duplicate object easy and accuracy, so in this paper discuss reverse engineering technology applied in making artificial bone more easily and reliable. In the past few years, it develop clinical reverse engineering for surgery applying, the bone 3D image can reconstruct by CT scanning image completely, after that use the rapid prototyping machine making full size graft bone. Compare with the 
traditional ways, the extra techniques of bone replenishment mode, the implant that had been produced can be accurate and looked nicely on the patients' bone gap. It also can cut down the recovery period of time after the surgeries. Therefore, our research will apply the technique of rapid prototyping powder and use bioceramic compound of PMMA, the quite common bone cement nowadays, and fine granule, the polymer of chitosan and TCP, as the prototyping material to discuss how to condense and mold bone replenishment for direct clinical usage with biochemistry composite powder material and rapid prototyping machine. [4-6].

Applied the RP stereo-shaped techniques, the powder material can match with related coagulant to directly coagulate into the mode to obtain the designing article solid mode. It is same with the conditions of bio-medical bone replenishing implant. During the bone replenishing clinical surgeries, the bone powder has to be related to the coagulant to go into a process of combining lacking bone replenishment.

As for the replenishment, the chosen rapid prototyping machine is Zcorp 402 3DP (figure 1.), a powder rapid prototyping machine, the convenience accession and the structure pattern after prototyping is a prototyping way of particular glue agglutination, which is different to the prototyping way of temperature fritting (as in figure 2.). With the images of the thesis, we use 3D image editorial software to the size of the testing replenishment with the ration of $1: 1$

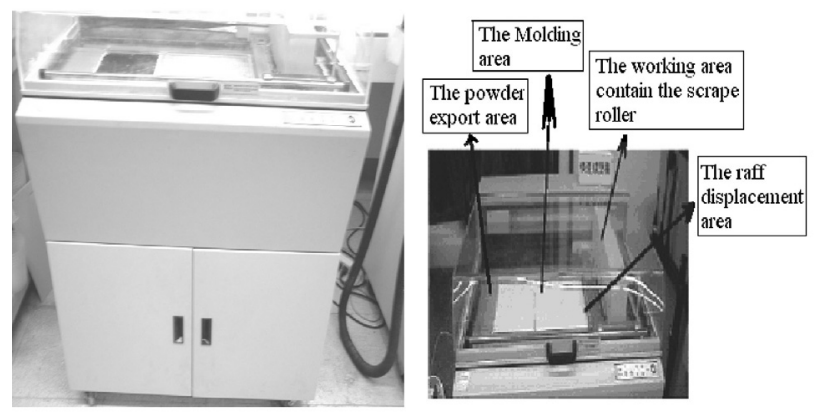

Fig. 1. The overview of prototyping platform for RP equipment.

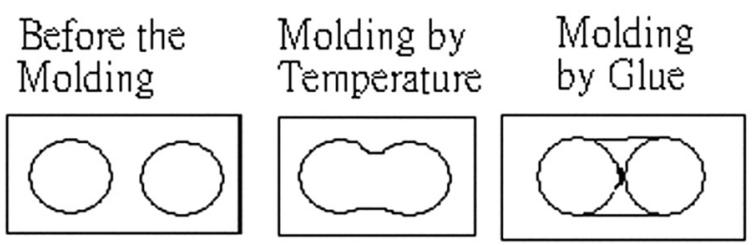

Fig. 2. The picture of molding by temperature and glue. to edit the image of the testing replenishment and transfer the STL file in order to provide the reading of Zcorp 3DP powder prototyping machine. The structure pattern of glue agglutination fays to the structure of bone concrete condensing prototyping. Besides, it's easy to make and can be the product of bone replenishment right after prototyping [3-5].

\section{MATERIAL AND METHOD}

Prepared biochemistry composite powder material (bone cement) and testing bone replenishment images can directly form the corporeal replenishment after imputed into R.P. equipment ( Zcorp 402 3DP). Once the ratio of the bone cement and forming glue are suitable then the image model of artificial implant bone sample will Inputting to R.P. equipment and can make artificial implant bone sample directly. The forming procedures are described as follows :

1. The shape of bone graft sample edited by the 3D images software. And the bone image will transforms into STL file.

2. Obtained and reviewed the bone graft sample image.

3. Set up the best forming parameters for Zcorp 402 3DP from user's guide reference.

4. Molding biochemistry bone powder into the powder type R.P. equipment.

5. Appling the powder type R.P. equipment shaping the bone graft sample.

The powder type R.P. machine Zcorp 402 is easy to be controlled on the quality and size. In this study, we mold bone cement into Zcorp 402. In this case we use the best parameters for Zcorp 402 3DP from reference, for example glue dose, molding thickness, the position furnishing and file parameter. This parameters correspond the adjustment for bone cement that the quality of mending one in order to improve shaping.

It is commercial prescription in glue, and it will make PMMA shaping and solidify after joining function. This kind of glue belongs to the high polymer material. The material will cause the other material dissolving during the forming process. The R.P. equipment that should keep the glue continues flowing, which contain the part of the material occupies by no dissolving. Among being at shaping it must avoid it is that glue flow through pipeline with R.P. direct contact of the part. This study is in Zcorp402 shower nozzle structure improvement that change the part of the shower nozzle of the first half into the material of ABS. It can avoid the dissolving to the shower nozzle of glue, and want to make the glue not pass the mechanical pipeline that packed the glue by the first half of the shower nozzle directly (Fig.3). And in the 


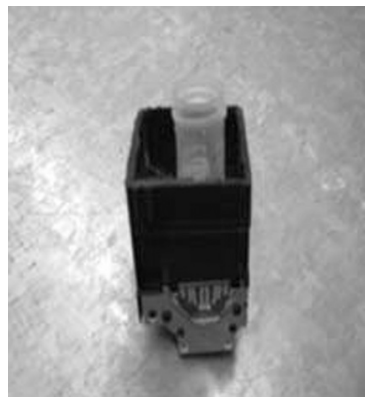

Fig. 3. The improvements of Z402 spray head and replace by ABS material.

course of shaping, consider its glue and send the pressure of the shower nozzle. So utilize the syringe to offer the supply glue of the pressure slightly by way of craft directly, this method can prevent the glue from touching the part within machinery. And the inside glue of the shaping machine still continues flowing fast in forming process, will not pass the shower nozzle and flow back to waste liquid directly. This can minimize this glue of loss to some parts of machinery, and can improve the shaping machine fast for growing the feasibility which cures material shaping of the bone graft. It can improve the shaping machine fast for growing the feasibility which cures material shaping of the bone graft. Improve the shower nozzle in the shower nozzle and forming process and install the way as following Figure 4.

The shape of bone graft sample edited by the 3D images software and link to the R.P. equipment's forming interface (Fig.5). This study using the bone cement material, which can apply it into powder base R.P. equipment directly and to make the samples as show in Fig.6. The first sample of this study make the graft block as the cylindrical shape which have $10 \mathrm{~mm}$ diameter and $25 \mathrm{~mm}$ of high. The sample will do the three points bending test and the compression intensity strength test. It is regular at three points bending test

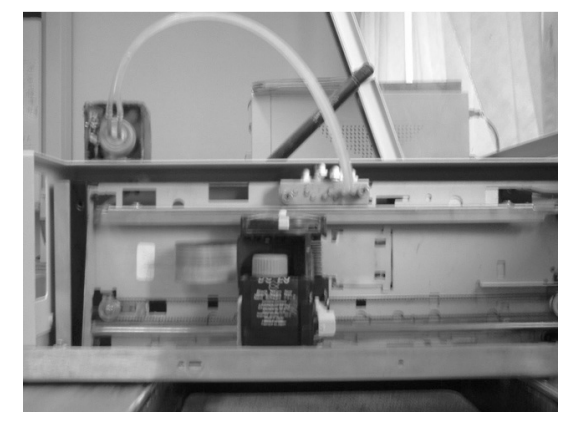

Fig. 4 .The improvement of PMMA glue supply unit (directly providing glue to spray head).

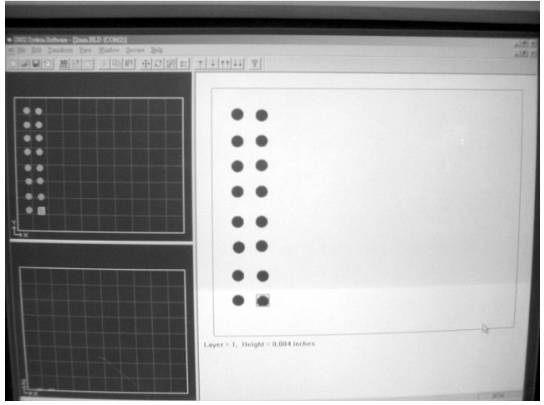

Fig. 5. The replenishing 3D image file in $R P$ equipment.

with the application of force of $10 \mathrm{~mm} / \mathrm{sec}$ in the middle direction until corrupter. In the compression intensity strength test at both end will apply $10 \mathrm{~mm} / \mathrm{sec}$ force to compress and until corrupter.

\section{RESULTS}

In this study that proportion of R.P. equipment can establishes a ratio to meet bone powder and glue. The implant bone graft molded by R.P. equipment that can form directly are suitable for the size, and do not need to making the molding else. This will have very great benefiting to the progressing greatly of medical quality. This research uses the replenishment, which was made from RP stereo-shaped techniques, the making process and sizes, and molding process did not have any changes in material characteristics. It can reach to the basic conditions with human implant.

Bone replenishment formed by mixing PMMA and fine granule of bone cement, the bone replenishment developed in the research are made by rapid prototyping machine, its parameters of mechanical test are shown in table I . Its strength is a little weaker than hand-pressed mixed bone powder replenishment, but it still fit the average bone strength that the body requires. According to each different body part, the required strength will be different. As for femur, it bears the strength of a half of the upper body. Materials with the compression larger than 60 MPa of bone strength or above in average, and the bending strength of 3 points are larger than $15 \mathrm{MPa}$ or above in average all can be use as a bone replacement.

As shown in table I, the bone replenishment developed in the research are made by rapid prototyping machine, therefore the strength is a little weaker than the strength of replenishment made in traditional way. The main reason for that is it bedded piled and it is not the traditionally made replenishment, but it still meets the requirement of human bone strength. 


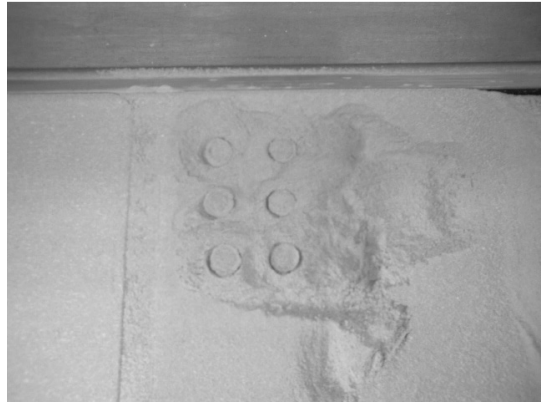

Fig. 6. The molded replenishments on a molding platform.

Table I. The mechanical properties of artificial bone graft sample

\begin{tabular}{|l|l|l|}
\hline $\begin{array}{l}\text { Bone graft } \\
\text { sample }\end{array}$ & $\begin{array}{l}\text { Maximum } \\
\text { compression } \\
\text { strength (MPa) }\end{array}$ & $\begin{array}{l}\text { Maximum } \\
\text { bending } \\
\text { strength (Mpa) }\end{array}$ \\
\hline $\begin{array}{l}\text { Hand-pressed } \\
\text { prototyping } \\
\text { with PMMA } \\
\text { individually }\end{array}$ & $90.6 \pm 0.7$ & $38 \pm 5.7$ \\
\hline $\begin{array}{l}\text { RP stereo- } \\
\text { shaped } \\
\text { techniques } \\
\text { with PMMA } \\
\text { bone powder }\end{array}$ & $71.4 \pm 0.5$ & $20.8 \pm 2.2$ \\
\hline
\end{tabular}

The mechanical properties and related characteristics of the replenishment made by this process can all satisfy the need of artificial bone implant. The only thing is the process of replenishment asepsis still needs to be done in the traditional way. If the production environment of the process of making replenishment in this research is aseptic, then it's able to produce germfree bone replenishment but the cost will also be high.

\section{CONCLUSION}

This study utilizes rapid prototyping technology (R.P.) to make the artificial implant bone graft which directly forming by the bone powder. Rapid prototyping machine not only can form bone replenishment directly from bone powder, but also make complicated curve replenishment for clinical use directly and conveniently. As shown in table I, the strength we tested can all fit the strength the human bones' need, and it can meet the need of the strength under weight bearing condition. This study is using the bone powder material, which can apply it into powder base R.P. equipment directly. It can get the perfect bone graft size and the relevant physical property can all meet the clinical demand of implant bone's characteristics, which offer another technique choice of forming artificial implant bone. This study also found the structure of bone graft is regularly bedded piled in the metal picture with lots of connected pores for cell growth, which is a better advantage. There will be a further discussion on the clinical biocompatibility in the future study.

\section{REFERENCES}

1. M. Kobayashi, T. Fujino, T. Kaneko,T.Kurihara, H.Chiyokura," Computer aided simulation surgery using a laser-curable resin model: Preoperative preparation of hydrixyapatice, for bone defect repair." In: Fujino T. Ed, Simulation and ComputerAided Surgery, Chichester, UK, John Wiley\& Sons, 129-135,1994.

2.Y. C. Tseng, "A study on material property and process parameter optimization for powder type rapid prototyping" National Kaohsiung Frist University of Science and Technology, Department of Mechanical and Automation Engineering, 2001.

3.M. Greul, T. Pintat and M. Greulich, "Rapid prototyping of functional metallic parts", Computers in Industry, Vol.18, Issue 1, December, 1995: 3-28.

4.K. D. V. Yarlagadda Prasad, C. Periklis, S. Vijay, "Feasibility studies on the production of electrodischarge machining electrodes with rapid prototyping and the electroforming process" , Journal of Materials Processing Technology, Vol.89, May 19, 1999: 231-237.

5.Zhang Wei, C. Leu Ming, Ji Zhiming and Yan Yongnian, "Rapid freezing prototyping eith water" , Materials and design, Vol.20, Issue 2-3, June 1, 1999:139-145.

6.S. P. Sun and J. S. Chen, "The application of fullscale 3D anthropometric digital model system on breast reconstruction of plastic surgeries", Biomedical Engineering Applications, Basis, and Communications, Oct. 2003. 